\title{
Inpatient rehabilitation outcome: a matter of diagnosis?
}

This article was published in the following Dove Press journal:

Neuropsychiatric Disease and Treatment

16 February 2013

Number of times this article has been viewed

\section{Maurizio Bejor' \\ Francesca Chiara Ramella \\ Elena Dalla Toffola' \\ Mario Comelli² \\ Matteo Chiappedi ${ }^{3}$}

'University of Pavia, Department of Surgical, Resuscitative, Rehabilitative and Transplant Sciences, Pavia, Italy; ${ }^{2}$ University of Pavia, Department of Brain Sciences, Medical Statistics Section, Pavia, Italy; ${ }^{3}$ Don Carlo Gnocchi ONLUS Foundation, Milan, Italy
Correspondence: Matteo Chiappedi Rehabilitation Unit, Santa Maria alle Fonti Medical Center, Don Carlo Gnocchi ONLUS Foundation, Viale Mangiagalli 52 - 27052 Salice Terme (PV), Italy

$\mathrm{Tel}+390383945612$

Fax +390383945 678

Email mchiappedi@dongnocchi.it
Background: Patients with comorbidities are becoming more and more common in Italian rehabilitative wards. These comorbidities are considered a major problem for inpatient rehabilitation, due to the fact that they cause longer lengths of stay, higher costs, and lower functional results.

Methods: To investigate the possible relationships between comorbidity, functional impairment, age, and type of discharge in patients hospitalized in postacute rehabilitation facilities, we planned an observational study. A total of 178 consecutive inpatients (average age: 78 years [range: 39-99]) from postacute rehabilitation facilities were recruited. Primary diagnosis, comorbidity rating (Cumulative Illness Rating Scale - Geriatric version, CIRS-G) and functional impairment score (Functional Independence Measure, FIM ${ }^{\mathrm{TM}}$ ) were evaluated at admission. The FIM ${ }^{\mathrm{TM}}$ rating was also assessed at hospital discharge.

Results: A total of 178 of the 199 enrolled patients completed the rehabilitation treatment (89.4\%). The average length of stay was $46 \pm 24$ days. CIRS-G showed an average comorbidity score for each patient of $4.45 \pm 1.69$. The average FIM ${ }^{\mathrm{TM}}$ rating was $79 \pm 24.88$ at admission, and $91.9 \pm 25.7$ at discharge. Diagnosis at admission (grouped according to the International Classification of Diseases 9-CM) seemed to correlate with functional results, since lower rehabilitative efficiency was obtained for patients who had a history of stroke.

Conclusion: The number and type of comorbidities (CIRS-G) in rehabilitation inpatients do not seem to affect functional outcomes of treatment. The determining factor for a lower level of functional recovery seems to be the diagnosis at admission.

Keywords: diagnosis, rehabilitation, inpatients, outcome

\section{Introduction}

In Italy in recent years, the number of hospitalizations and the average length of stay (LOS) for rehabilitation have greatly increased; more and more patients are referred to rehabilitative wards and, at the same time, their baseline clinical condition is progressively worsening. A recent official document from the Italian Ministry of Health recognizes this fact and points out the need to develop new strategies to increase ward efficiency, thus avoiding the progressive increase (especially in terms of cost) in this sector. ${ }^{1}$

In Lombardy, the northern Italian region where one-sixth of the entire Italian population lives (more than 10 million inhabitants), the rehabilitation centers are closely concerned with this problem. In response, its health authority has developed a fairly complex public health service that, as in most western European countries, aims to cover the whole population. 
Since the early 2000s, these centers have been planned with two main ward types. The first, called "riabilitazione specialistica" (specialist rehabilitation wards), is intended to offer more intensive rehabilitative activity to patients in either relatively fair general conditions, or with complex problems such as traumatic brain injuries. The second, called "riabilitazione generale geriatrica" (general and geriatric rehabilitation wards), is intended to offer less intensive, but prolonged rehabilitative activity mainly for elderly patients. It is also suited for those patients whose general health conditions have reduced their functional capacity in vital domains such as cardiovascular or respiratory function, which do not allow them to tolerate an intensive rehabilitative treatment. ${ }^{2}$

Even if the "riabilitazione specialistica" have higher reimbursement fees than the "riabilitazione generale geriatrica," it has been shown that clinical data such as diagnosis, age, level of impairment, and number of comorbidities are unable to differentiate between patients seen in the different ward types. ${ }^{3}$ This could mean that these different ward types are not used as intended according to current definitions, or it could mean that all patients seen in rehabilitative wards are very similar, and it is therefore hard to create and respect different pathways.

Generally speaking, inpatient rehabilitation can be described as a multidisciplinary intervention that aims at increasing a patient's possibility to perform daily living activities. Treatment options include not only treatments directed to the patient (eg, motor therapy, speech therapy, occupational therapy), but also interventions particular to his/ her environment (eg, prescription of orthoses, specifically adapted tools, or activation of welfare support).

Comorbidity can be defined as a clinical condition that is already present at the onset of the main disease for which a patient is admitted to the hospital, and that affects the clinical course of the patient. ${ }^{4}$ In most cases, comorbidity in hospitalized patients is evaluated using clinical data, as well as using the results of laboratory and diagnostic tests. ${ }^{5}$

Several indices have been used to evaluate comorbidity in the epidemiologic and clinical fields. A widely used index is the Cumulative Illness Rating Scale (CIRS), which has been shown to be a good indicator of the health status and outcome (risk of death) of hospitalized patients. ${ }^{6}$ The CIRS is structured into 13 domains related to different body systems/ functions; each of these domains is evaluated by assigning an increasing severity rating ranging from 1 to $5 .^{7}$

In 1992, a specific adjustment to the CIRS was proposed for use on the geriatric population and was named CIRS-G. This index includes 14 disease categories (extrapolating diabetes as a specific category). The CIRS-G has been shown to be valid by comparing CIRS-G ratings assigned by clinicians with those based on autopsy findings (gold standard). ${ }^{8}$

When the severity scores assigned to the pathologies contained within the first 13 CIRS-G systems are processed, we obtain two indices: the severity index (the average rating) and the comorbidity index (the number of categories whose score is equal to or greater than 3). Psychiatric and behavioral disorders are included as a separate domain, but are not considered in the computation of either the severity or the comorbidity indices.

The associated pathologies observed in patients hospitalized in more intensive rehabilitation facilities ("riabilitazione specialistica") may cover several areas with different degrees of severity. ${ }^{9}$ In the field of rehabilitation, the presence of five or more comorbidities has been shown to contribute to lower functional recovery, ${ }^{10}$ to less effective rehabilitation, ${ }^{11}$ to longer LOSs, ${ }^{12}$ to a higher level of subsequent hospitalization, ${ }^{13}$ and to higher hospital costs. ${ }^{14}$

The aim of this study is to investigate which factors influence the functional outcome of inpatients seen in rehabilitation facilities. According to clinical experience, we hypothesized that diagnosis on admission (ie, the reason for sending the patients for rehabilitation) could be the main prognostic factor, although in the scientific literature the role of comorbidity is greatly emphasized.

\section{Materials and methods}

We enrolled 199 patients (56 males (28.1\%), 143 females (71.9\%); average age, $78.2 \pm 11.3$ years) consecutively admitted in 2010 to a specialist rehabilitation facility ("Santa Maria alle Fonti" Medical Center, Salice Terme, Pavia, Italy), where an interdisciplinary team had established an individualized postacute rehabilitation program. These patients had been discharged from surgical and medical acute care units after treatment was considered to be adequate given that it stabilized vital functions and allowed the patients to attend rehabilitation programs. Patients admitted directly from their homes were excluded because it was not possible to verify their initial health conditions (ie, we could not exclude a priori an acute and eventually life-threatening disorder). Moreover, patients with a Folstein's Mini Mental State Examination score $<20$ were excluded as they would not have been able to collaborate with a rehabilitation program. ${ }^{15}$

All patients were informed about the procedures and purposes of the investigation, and they provided written informed consent. The study was conducted following recommendations from the Helsinki Declaration. 
At admission we collected the following data: primary reason for admission according to the International Classification of Diseases (ICD)-9-CM classification system, CIRS-G indexes (based on medical history, clinical examination, and laboratory findings), and functional impairment as assessed by the Functional Independence Measure (FIM ${ }^{\mathrm{TM}}$ ) scale (UB Foundation Inc., Buffalo, NY, USA).

The FIM $^{\mathrm{TM}}$ scale is a widely used tool; a trained clinician has to rate the level of independence of the patient in performing daily activities (ranging from 1, completely dependent, to 7 , completely independent) ${ }^{16}$ Since 18 items are included (13 motor activities and five cognitive capacities), the score can range from 18 to $126 .{ }^{10}$ The assessment in this study was conducted by a trained clinician.

At discharge we collected the LOS, the type of discharge (regular, death, transfer to acute care unit, voluntary patient discharge), the efficacy (defined as FIM $^{\mathrm{TM}}$ score at discharge - FIM ${ }^{\mathrm{TM}}$ score at admission), ${ }^{17}$ and the efficiency (defined as efficacy/LOS) of the rehabilitative treatment.

A multiple regression model was fitted to the functional improvement, including comorbidity, admission diagnosis type, age, and FIM ${ }^{\mathrm{TM}}$ rating at admission as explanatory variables. All of the explanatory variables were included simultaneously in one single step. No serious deviations from normality of the residuals were observed.

LOS and efficiency were found not to be distributed normally (Kolmogorov-Smirnov test: $P<0.001$ ). Differences in these parameters according to the diagnostic group were therefore analyzed using the Kruskal-Wallis test.

The computations were executed using the package $\mathrm{R}(\mathrm{R}$ Development Core Team, Wien, Austria). ${ }^{18}$

\section{Results}

A total of 178 of the 199 patients initially enrolled in the study $(89.4 \%)$ completed the whole treatment, nine $(4.5 \%)$ died during hospitalization, nine $(4.5 \%)$ were transferred to acute care units following clinical complications, and three $(1.6 \%)$ voluntarily discontinued treatment (details are given in Table 1). Statistical analysis included only patients regularly discharged.
The average hospitalization length was $46 \pm 24$ days. The average FIM ${ }^{\mathrm{TM}}$ rating was $79 \pm 24.88$ at admission and $91.9 \pm 25.7$ at discharge.

We divided the admission diagnoses of enrolled patients into five main diagnostic groups on the basis of ICD-9-CM criteria: 66 patients $(37.1 \%)$ were admitted following a physical trauma, 35 (19.7\%) with musculoskeletal disorders (eg, arthritis), 34 (19.1\%) with cerebrovascular diseases (strokes), 20 (11.2\%) with infectious diseases (involving the central nervous system, such as Guillain Barré), and $23(12.9 \%)$ with degenerative diseases of the nervous system or sense organs (including Parkinson's disease and multiple sclerosis).

The average value of CIRS-G scores was $4.45 \pm 1.69$ comorbidities (range: $0-10)$, of which 155 (17.71\%) were related to the cardiocirculatory system, $126(14.40 \%)$ were caused by arterial hypertension, $114(13.0 \%)$ were related to the nervous system or sense organs, $74(8.46 \%)$ were related to the musculoskeletal system, $55(6.29 \%)$ were trauma disorders, 54 (6.17\%) were mental disorders, 48 (5.49\%) were related to the digestive system, $40(4.57 \%)$ were related to the genitourinary system, and 38 (4.34\%) were diabetes.

In the multiple regression model, the comorbidity index did not appear to influence the functional outcome; its effect (adjusted according to admission diagnosis type, age, and FIM $^{\mathrm{TM}}$ rating at admission) turned out to be 0.14 points for each additional disease category involved $(P=0.80$, confidence interval [CI]: -0.96 to 1.24 ).

The admission diagnosis was significantly related to the functional outcome; patients with infectious diseases involving the central nervous system were estimated to improve by 9.51 ( $P=0.009$; CI: $2.44-16.58)$ FIM $^{\mathrm{TM}}$ points more than those with a stroke; the same applied to those with limb fractures (11.23 points; $P<0.001$; CI: 6.04-16.41) and musculoskeletal disorders (6.83 points; $P=0.03$; CI: $0.78-12.88)$. The differences were not significant for patients with neurodegenerative disorders (3.56 points; $P=0.29$; CI: -3.18 to 10.29 ), although we could not rule out an insufficient powering effect since this was one of the diagnostic groups with a smaller number of patients. Age and

Table I Study population (data given as mean \pm SD)

\begin{tabular}{lcllc}
\hline Patients (I 99; I 00\%) & Mean age & FIM ${ }^{\text {TM }}$ at admission & \multicolumn{1}{c}{ CIRS-G comorbidity index } & CIRS-G severity index \\
\hline Regularly discharged (I78; 89.4\%) & $77.54 \pm 29.34$ & $81.12 \pm 23.96$ & $4.48 \pm 1.73$ & $0.7 \pm 0.27$ \\
Transferred (9; 4.5\%) & $81.39 \pm 12.8$ & $71.33 \pm 26.35$ & $4.11 \pm 1.17$ & $0.64 \pm 0.2$ \\
Deceased (9; 4.5\%) & $88.2 \pm 4.43$ & $50.78 \pm 25.34$ & $4.33 \pm 1.32$ & $0.56 \pm 0.29$ \\
Voluntarily discharged (3; I.6\%) & $75.82 \pm 4.27$ & $70.00 \pm 10.54$ & $1.67 \pm 1.47$ & $3.00 \pm 2.65$ \\
\hline
\end{tabular}

Abbreviations: SD, standard deviation; FIM, Functional Independence Measure; CIRS-G, Cumulative Illness Rating Scale - Geriatric version. 
FIM $^{\mathrm{TM}}$ rating at admission turned out to have only marginally significant negative effects on FIM $^{\mathrm{TM}}$ score at discharge (both $P$-values $=0.07)$. The whole model and results are depicted in Table 2.

Although the average LOS was higher in the group of patients with limb fractures, this was not statistically significant. In contrast, efficiency was significantly lower in the group of patients suffering from strokes compared to the others $(P=0.023)$. Since the average LOS was not significantly different between groups, this was explained by a lower increase of the FIM ${ }^{\mathrm{TM}}$ rating for patients with strokes compared to those with other diagnoses.

\section{Discussion}

In our study, the outcome of patients who received rehabilitative treatment was significantly influenced by the main diagnosis on admission.

Previous studies reported lower functional recovery and reduced rehabilitation effectiveness in patients with more comorbidities, ${ }^{10,11}$ whereas in another study reduced functional recovery or deterioration were significantly correlated with a greater functional impairment at admission. ${ }^{19}$

Our findings seem instead to indicate that the major factor influencing the functional outcome of inpatient rehabilitation is the main diagnosis on admission; patients who had had a stroke improved less and more slowly than those with other disorders, with a minor role played by comorbidities and age.

In contrast with previous studies, ${ }^{14}$ the LOS of our patients did not seem to directly affect the outcome of the rehabilitative treatment, even though our data confirmed that the level of efficiency of rehabilitation (ie, the average improvement in the FIM ${ }^{\mathrm{TM}}$ score per day of hospitalization) could differ according to the main diagnosis.

While we acknowledge the fact that it is necessary to continue research in this field, this study shows that inpatient rehabilitation can be performed in almost any patient, but it probably needs to be tailored according to several factors, including the patient's main diagnosis.

This is also an important result for administrative purposes. Given the relevant role played by the main diagnosis at admission, it is possible that rehabilitation wards could be better divided according to their possibility to offer highly specialized and cost-effective rehabilitation for some specific disorders (eg, cerebrovascular diseases); patients could then be transferred to the most appropriate rehabilitative setting. The reimbursement system should also be improved to follow the evidence of a longer average LOS and of a more intensive and specific intervention for some disorders.

An unexpected finding was the fact that age at admission did not correlate with the results of rehabilitation, nor did it correlate with the LOS. ${ }^{20}$ It is possible, however, that the effect of age noted in previous studies was a consequence of an increased incidence of some disorders in older patients (eg, cerebrovascular disorders) or of a higher level of significant comorbidities. $^{21}$

\section{Conclusion}

Our findings seem to indicate that the diagnosis at admission is a major factor influencing the functional outcome of inpatients seen in rehabilitative wards. This could have implications for the definition of specific care pathways, and also for the organization (and the reimbursement) of rehabilitative wards.

Our study has two relevant limitations. First, its observational design does not allow any definite conclusion to be drawn, as variables not included in the assessment might have interfered with our study. Second, it was conducted in a specific context (Lombardy, a region of Italy); therefore, findings may not be directly generalizable to other countries.

\section{Disclosure}

The authors report no conflicts of interest in this work. The authors did not receive grants or funding for this paper.

Table 2 Multiple regression model

\begin{tabular}{llll}
\hline & Coefficient & $P$-value & Confidence interval \\
\hline Intercept & 20.80 & 0.02 & $3.43-38.17$ \\
Physical trauma (limb fractures) & 11.23 & $<0.001$ & $6.04-16.41$ \\
Musculoskeletal disorders & 6.83 & 0.03 & $0.78-12.88$ \\
Infectious diseases (involving the CNS) & 9.51 & 0.009 & $2.44-16.58$ \\
Neurodegenerative disorders & 3.56 & 0.29 & -3.18 to 10.29 \\
Age at admission & -0.16 & 0.07 & -0.34 to 0.01 \\
Comorbidity index & 0.14 & 0.80 & -0.96 to 1.24 \\
FIM $^{\text {TM }}$ at admission & -0.08 & 0.07 & -0.16 to 0.01 \\
\hline
\end{tabular}

Abbreviations: CNS, central nervous system; FIM, Functional Independence Measure. 


\section{References}

1. Ministero Della Salute. Piano D'indirizzo per la Riabilitazione. Rome: Ministero della Salute, Dipartimento Della Qualita. Available online: http://www.simferweb.net/blog/wp-content/uploads/2011/02/604piano-indirizzo-riabilitazione.pdf. Accessed October 31, 2012. Italian.

2. Regione Lombardia. The 2007-2009 Lombardy Region Social Services and Health Care Plan [webpage on the Internet]. Lombardy: Regione Lombardia. Available from: http://www.sanita.regione.lombardia.it/. Accessed October 31, 2012. Italian.

3. Bejor M, Pezzoli A, Dalla Toffola E, Chiappedi M. Main diagnosis, comorbidity and functional impairment at admission in the rehabilitative wards of Lombardy region. A survey on 6,671 patients. Eur J Phys Rehabil Med. 2010;46(4):609-611.

4. Powell H, Lim LL, Heller RF. Accuracy of administrative data to assess comorbidity in patients with heart disease. An Australian perspective. J Clin Epidemiol. 2001;54(7):687-693.

5. Rozzini R, Frisoni GB, Ferrucci L, et al. Geriatric Index of Comorbidity: validation and comparison with other measures of comorbidity. Age Ageing. 2002;31(4):277-285.

6. Giaquinto S, Palma E, Maiolo I, et al. Importance and evaluation of comorbidity in rehabilitation. Disabil Rehabil. 2001;23(7):296-299.

7. Miller MD, Paradis CF, Houck PR, et al. Rating chronic medical illness burden in geropsychiatric practice and research: application of the Cumulative Illness Rating Scale. Psychiatry Res. 1992;41(3):237-248.

8. Conwell Y, Forbes NT, Cox C, Caine ED. Validation of a measure of physical illness burden at autopsy: the Cumulative Illness Rating Scale. J Am Geriatr Soc. 1993;41(1):38-41.

9. Bellelli G, Magnifico F, Trabucchi M. Outcomes at 12 months in a population of elderly patients discharged from a rehabilitation unit. J Am Med Dir Assoc. 2008;9(1):55-64.

10. Stineman MG, Maislin G, Williams SV. Applying quantitative methods to the prediction of full functional recovery in adult rehabilitation patients. Arch Phys Med Rehabil. 1993;74(8):787-795.
11. Dromerick A, Reding M. Medical and neurological complications during inpatient stroke rehabilitation. Stroke. 1994;25(2):358-361.

12. Ceder L, Thorngren KG, Wallden B. Prognostic indicators and early home rehabilitation in elderly patients with hip fractures. Clin Orthop Relat Res. 1980;(152):173-184.

13. Rodriguez GS, Goldberg B. Rehabilitation in the outpatient setting. Clin Geriatr Med. 1993;9(4):873-881.

14. Patrick L, Knoefel F, Gaskowski P, Rexroth D. Medical comorbidity and rehabilitation efficiency in geriatric inpatients. J Am Geriatr Soc. 2001;49(11):1471-1477.

15. Folstein MF, Folstein SE, McHugh PR. "Mini-mental state". A practical method for grading the cognitive state of patients for the clinician. J Psychiatr Res. 1975;12(3):189-198.

16. Stineman MG, Jette A, Fiedler R, Granger C. Impairment-specific dimensions within the Functional Independence Measure. Arch Phys Med Rehabil. 1997;78(6):636-643.

17. Johnston MV, Wood KD, Fiedler R. Characteristics of effective and efficient rehabilitation programs. Arch Phys Med Rehabil. 2003;84(3):410-418.

18. R Development Core Team. R: A language and environment for statistical computing [homepage on the Internet]. Wien, Austria: R Development Core Team. Available from: http://www.R-project. org. Accessed March 30, 2012.

19. Lew HL, Lee E, Date ES, Zeiner H. Influence of medical comorbidities and complications on FIM change and length of stay during inpatient rehabilitation. Am J Phys Med Rehabil. 2002;81(11):830-837.

20. Semel J, Gray JM, Ahn HJ, Nasr H, Chen JJ. Predictors of outcome following hip fracture rehabilitation. P M R. 2010;2(9):799-805.

21. Jang HJ, Park J, Shin HI. Length of hospital stay in patients with spinal cord injury. Ann Rehabil Med. 2011;35(6):798-806.
Neuropsychiatric Disease and Treatment

\section{Publish your work in this journal}

Neuropsychiatric Disease and Treatment is an international, peerreviewed journal of clinical therapeutics and pharmacology focusing on concise rapid reporting of clinical or pre-clinical studies on a range of neuropsychiatric and neurological disorders. This journal is indexed on PubMed Central, the 'PsycINFO' database and CAS.

\section{Dovepress}

The manuscript management system is completely online and includes a very quick and fair peer-review system, which is all easy to use. Visit http://www.dovepress.com/testimonials.php to read real quotes from published authors. 